\title{
Tandem mass spectrometry identification and LC-MS quantification of intact cytokinin nucleotides in K-562 human leukemia cells
}

\author{
Tibor Béres • Marek Zatloukal • Jiří Voller • Percy Niemann • \\ Marie Christin Gahsche • Petr Tarkowski • Ondřej Novák • Jan Hanuš • \\ Miroslav Strnad • Karel Doležal
}

Received: 10 May 2010 /Revised: 16 July 2010 / Accepted: 11 August 2010 / Published online: 7 September 2010

(C) Springer-Verlag 2010

\begin{abstract}
We describe here a new reversed-phase highperformance liquid chromatography with mass spectrometry detection method for quantifying intact cytokinin nucleotides in human K-562 leukemia cells. Tandem mass spectrometry was used to identify the intracellular metabolites (cytokinin monophosphorylated, diphosphorylated, and triphosphorylated nucleotides) in riboside-treated cells. For the protein precipitation and sample preparation, a trichloroacetic acid extraction method is used. Samples are then back-extracted with diethyl ether, lyophilized, reconstituted, and injected into the LC system. Analytes were quantified in negative selected ion monitoring mode using a single quadrupole mass spectrometer. The method was
\end{abstract}

T. Béres $\cdot$ M. Zatloukal $\cdot$ J. Voller $\cdot$ P. Tarkowski $\cdot$ O. Novák •

M. Strnad $\cdot$ K. Doležal $(\bowtie)$

Laboratory of Growth Regulators,

IEB AS CR and Palacký University,

Šlechtitelů 11,

78371 Olomouc, Czech Republic

e-mail: karel.dolezal@upol.cz

P. Tarkowski

Department of Biochemistry, Faculty of Science,

Palacký University,

Šlechtitelů 11,

78371 Olomouc, Czech Republic

P. Niemann - M. Christin Gahsche

BIOLOG Life Science Institute,

Flughafendamm 9a,

28199 Bremen, Germany

J. Hanuš

Institute of Experimental Botany AS CR,

Vídenská 1083,

14220 Prague 4, Czech Republic validated in terms of retention time stabilities, limits of detection, linearity, recovery, and analytical accuracy. The developed method was linear in the range of $1-1,000 \mathrm{pmol}$ for all studied compounds. The limits of detection for the analytes vary from 0.2 to $0.6 \mathrm{pmol}$.

Keywords Cytokinins $\cdot$ Nucleotides $\cdot$ HPLC .

Mass spectrometry $\cdot \mathrm{K}-562$

\section{Introduction}

Cytokinins (CKs) are an important group of purine-based plant hormones that are involved in the regulation of cell division and differentiation in plants [1]. Studies of purine metabolism show they exist as free bases, glycosides, and nucleotides. Their biosynthetic pathway, as well as metabolism in plants, has been studied for decades, but the early steps of their biosynthesis were not fully elucidated until recently. In 2001, an in silico search of the Arabidopsis genome revealed nine putative genes (AtIPT1-AtIPT9) encoding isopentenyl transferases [2, 3]. Subsequent experiments with recombinant AtIPT4 enzyme showed that it preferentially utilizes adenosine triphosphate (ATP) and adenosine diphosphate (ADP) over adenosine monophosphate (AMP), indicating that the first cytokinins in this biosynthetic pathway are diphosphate and triphosphate isopentenyl adenosines (iPR) [2], respectively. Subsequent side-chain hydroxylation leads to the formation of cis- or trans-zeatin riboside diphosphate and triphosphate. In accordance to this recent development in cytokinin biosynthesis research [2,3], availability of the quantification method capable of direct determination of different cytokinin 
nucleotide species would be crucial for detailed characterization of individual isopentenyl transferases in model plants as well as in important crops. Detailed description of substrate specificities (as well as other important biochemical characteristics) of this key cytokinin biosynthetic enzyme group has never been performed satisfactorily so far because of lack of appropriate analytical method. This is not possible using routine methods of cytokinin analysis currently available [4].

Surprisingly, aside from their roles in the growth regulation of higher plants, CK nucleotides seem to function as triggers for the apoptosis of cancer cells treated with corresponding cytokinin ribosides. Naturally occurring cytokinin ribosides and some of their synthetic analogs show cytotoxicity towards various human cancer cell lines $[5,6]$. In several cases, it has been shown that intracellular phosphorylation is essential for inducing cell death $[7,8]$. In addition, Mlejnek and Doležel showed that CK monophosphates accumulate in HL-60 cells, although the amounts of diphosphate and triphosphates present (if any) were below the detection limits of the method used [8].

Adenosine nucleotides and their synthetic analogs are usually poorly retained on standard reversed-phase (RP) columns due to their high polarity. Enzymatically cleaving the phosphate groups [9], or non-enzymatically hydrolyzing them [10], prior to high-performance liquid chromatography (HPLC) analysis overcomes the problem of poor retention, but the individual nucleotides cannot then be identified.

Intact nucleotides are often identified using ion-pairing chromatography, with cations as counter-ions. Quaternary ammonium salts are often used for this purpose, as they are positively charged over a wide $\mathrm{pH}$ range. Their low volatility complicates their use with MS detection systems, but this problem can be partially overcome using microbore or capillary columns and a coaxial sheath gas [11].

Alternatively, MS compatibility of the mobile phase can be achieved using volatile dialkylamines or trialkylamines as ion-pairing agents [12-14]. Another possibility for nucleotide identification is to use weak anion exchange chromatography [15]. This approach has been successfully applied for determining the intracellular metabolites of the 2-chloro-2'-deoxyadenosine analog cladribine.

Three basic approaches of CK nucleotide analysis have been applied in previous studies: (1) enzymatic cleavage of the ribose phosphate/s moiety/ies followed by highperformance liquid chromatography with mass spectrometry (HPLC-MS) [9], (2) derivation of the free hydroxyl groups of CK nucleotides by carboxylic acid anhydride [16], and (3) intact monophosphorylated nucleotide analysis by capillary electrophoresis with tandem mass spectrometry [17]. These methods provide information about total CK nucleotide contents or are focused on identifying the monophosphates only. Here, we present (to our knowledge) the first report of the direct analysis of intact CK nucleotides (monophosphates, diphosphates, and triphosphates) by HPLC-MS. The usefulness of this method was demonstrated by its application to quantify cytokinin nucleotides in a human cancer cell line (K-562) treated with iPR.

\section{Experimental}

\section{Chemicals}

All reagents were of analytical grade or the best grade available from commercial suppliers. ${ }^{2} \mathrm{H}_{5}$-trans-zeatin riboside-5'-monophosphate was purchased from OlChemIm (Olomouc, Czech Republic). 6-Chloropurine-9- $\beta$-D-riboside5'-O-diphosphate and 6-chloropurine-9- $\beta$-D-riboside-5'- $O$-triphosphate was purchased from BIOLOG (Bremen, Germany). The synthesis of isopentenyl adenosine diphosphate and triphosphate was published previously [18]. Other standards were prepared as described below (for chemical structures, see Table 1). Methanol and $n$-propanol were purchased from Lachner; $N$-ethyl- $N, N$-diisopropyl amine $(98 \%+)$ was from Sigma-Aldrich; 6-chloropurine-9- $\beta$-Driboside-5'-O-monophosphate $(98 \%+)$ was from OlChemIm. 3-Methylbut-2-ene-1-yl amine was prepared according to Summons et al. [19]. (E) and (Z)-4-Amino-2-methylbut-2ene-1-ol hemioxalate (95\%), as well as trans-zeatin(D5)amine hemioxalate, was obtained from Dr. Jan Hanuš, Institute of Experimental Botany ASCR, Prague. HPLCgradient-grade methanol, formic acid, acetic acid, and ammonium hydroxide were from Sigma-Aldrich (St. Louis, MO, USA). Deionized (Milli-Q) water was from Simplicity 185 (Millipore, Bedford, MA, USA). Trichloroacetic acid and diethyl ether (p.a.) were from Lachner (Brno, Czech republic).

\section{Instrumentation}

The HPLC-MS quantification method was validated, and the synthesized monophosphate standards were characterized using an Alliance 2695 Separations Module (Waters, Milford, MA, USA) linked simultaneously to a 996 photo diode array detector (PDA) and a ZMD 2000 single quadrupole mass spectrometer equipped with an electrospray interface (Micromass, Manchester, UK). An Acquity UPLCTM System (Waters, Milford, MA, USA), including a binary solvent manager, sample manager, and 2996 PDA detector, combined with a Micromass Quattro Micro ${ }^{\mathrm{TM}}$ API (Micromass, Manchester, UK) was used for HPLC-MS/MS identification experiments. Data were processed by MassLynx software (Data Handling System for Windows, version 4.0, Micromass, Milford, MA, USA). 
Table 1 Structures, names, and abbreviations of CK nucleotides used in this study

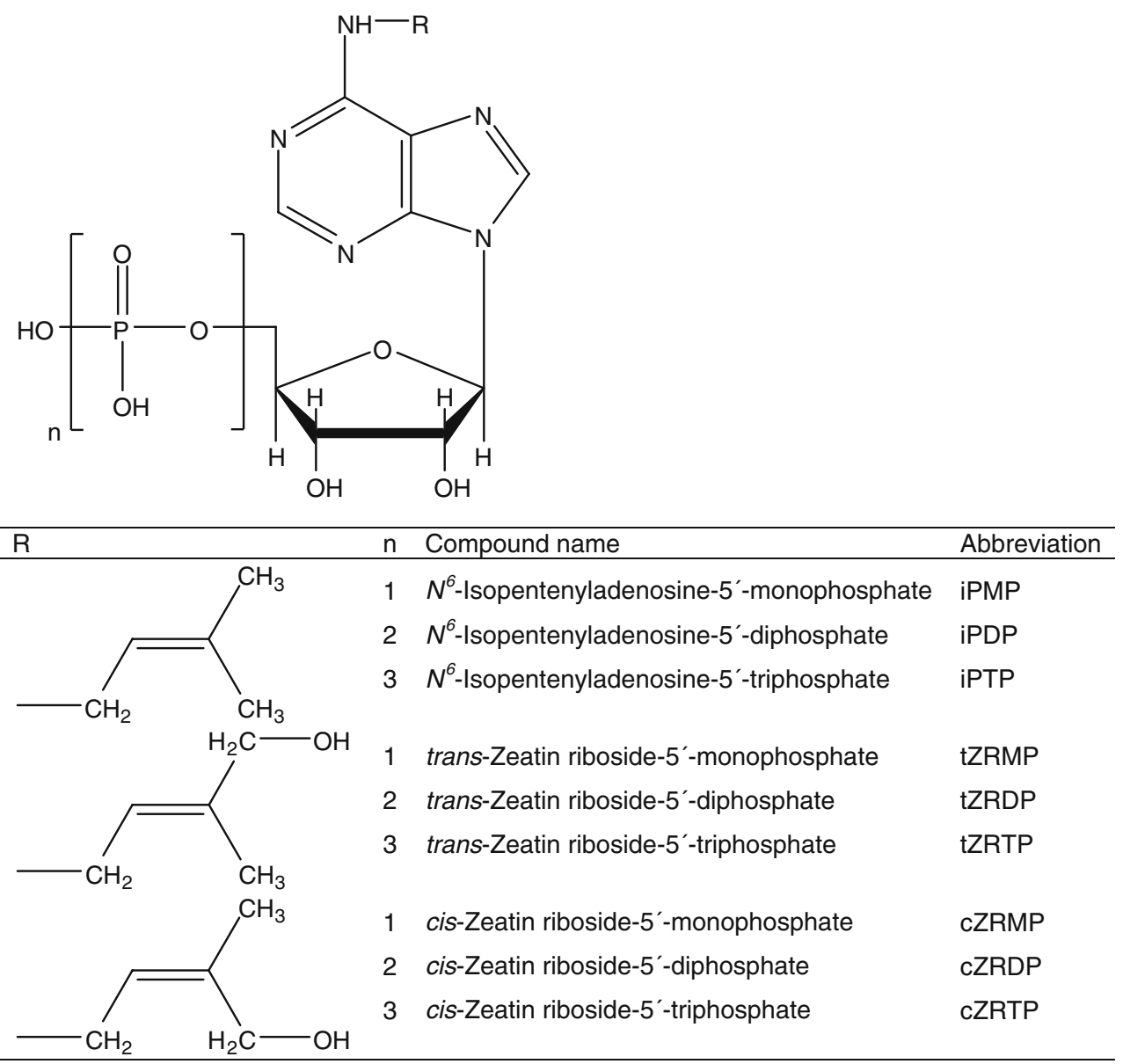

The analytical HPLC system for the CK diphosphate and triphosphate purity and product formation progress measurements, as well as the preparative HPLC system for product purification, consisted of a L7100 pump, a L7400 variable wavelength UV detector, and a D 7500 Integrator (MerckHitachi, Darmstadt, Germany).

An Esquire 6000 spectrometer (Bruker, Bremen, Germany) was used for the mass spectrometry of the prepared diphosphates and triphosphates.

Nuclear magnetic resonance (NMR) spectra of the monophosphates were measured using a Bruker Avance DRX 300 spectrometer operating at a frequency of $300.13 \mathrm{MHz}(1 \mathrm{H})$ and a temperature of $297 \mathrm{~K}$. Samples were prepared by dissolving the compounds in deuterated dimethyl sulfoxide (DMSO-D6), and tetramethyl silane was used as an internal standard.

Preparation of standards

\section{Monophosphates}

The (E/Z)-zeatin-9- $\beta$-D-riboside-5'-monophosphates and $\mathrm{N}^{6}$-isopentenyladenosine-5'-monophosphate (iPMP) were prepared by nucleophilic substitution of 6-chloropurine-9$\beta$-D-riboside-5'-monophosphate disodium salt dihydrate $(0.5 \mathrm{mmol})$ with $0.6 \mathrm{mmol}$ of the corresponding amine (E/Z)-4-hydroxy-3-methylbut-2-enyl amine or 3,3-dimethylallylamine, in methanol or $n$-propanol. $N, N$-Diisopropyl- $N$ ethyl amine $(5 \mathrm{mmol})$ was then added dropwise. The reaction was carried out in a glass Ace pressure tube (Sigma-Aldrich; $35 \mathrm{ml}$ ) in which an argon or nitrogen atmosphere was strictly maintained to avoid possible oxidation. The temperature of the reaction mixture was kept between 90 and $100{ }^{\circ} \mathrm{C}$ for 6-12 h. Subsequently, the solvent was removed by vacuum distillation using a rotary vacuum evaporator. The glassy residue was purified by flash column RP C18 chromatography with a mobile phase of $15 \%$ methanol in water. The product was purified by crystallization from 2-propanol. Details of reaction conditions, yields, and purities are given in Table 2.

\section{Diphosphates and triphosphates}

All $\mathrm{N}^{6}$-substituted adenosine-5'-O-diphosphates and triphosphates used in the present study were synthesized by reacting the corresponding 6-chloropurine-9- $\beta$-D-riboside-5'-O- 
Table 2 Reaction conditions and $\mathrm{m} / \mathrm{z}$ of deprotonated quasimolecular ions (ESI ${ }^{-} \mathrm{MS}$ ) of the prepared compounds

\begin{tabular}{lcccc}
\hline Compound & {$[\mathrm{M}-\mathrm{H}]^{-}$} & Reaction temperature $\left({ }^{\circ} \mathrm{C}\right)$ & Yield (\%) & HPLC purity (\%) \\
\hline iPMP & 414.3 & 100 & 82 & 95 \\
tZRMP & 430.1 & 100 & 80 & 95 \\
tZRDP & 510.1 & 40 & 38 & $>99$ \\
tZRTP & 590.0 & 40 & 34 & $>99$ \\
cZRMP & 430.1 & 90 & 50 & 95 \\
cZRDP & 510.1 & 40 & 40 & $>99$ \\
cZRTP & 590.0 & 40 & 34 & $>99$ \\
D5-tZRDP & 515.1 & 40 & 36 & $>99$ \\
D5-tZRTP & 595.1 & 40 & 40 & $>99$ \\
\hline
\end{tabular}

diphosphate or triphosphate precursor, as a triethylammonium salt, with various cytokinin side-chain amines under alkaline aqueous conditions. Briefly, $50 \mu \mathrm{mol}$ of 6-chloropurine nucleotide (6-Cl-PuDP and 6-Cl-PuTP, respectively), triethylammonium salt, was dissolved in $3 \mathrm{ml} \mathrm{H}_{2} \mathrm{O}$ and $1.5 \mathrm{ml}$ ethanol in a reaction tube with a screw cap. After adding $100 \mu \mathrm{mol}$ (two equivalents) of amine, the $\mathrm{pH}$ was adjusted to 11 with $2 \mathrm{M} \mathrm{NaOH}$, and the tube was gently shaken at $40{ }^{\circ} \mathrm{C}$ in a thermo mixer. For reaction condition details, see Table 2. The progress of product formation was monitored by analytical HPLC. The reaction was quenched by adjusting the $\mathrm{pH}$ to 7.6 using formic acid and subsequent cooling to $-70{ }^{\circ} \mathrm{C}$. The product was purified by preparative HPLC with DEAE sepharose as the stationary phase and $300 \mathrm{mM}$ triethylammonium bicarbonate $(\mathrm{pH} 8.0)$ as the mobile phase. The product-containing fractions were collected, concentrated under reduced pressure, and desalted by preparative HPLC on reversed-phase silica gel with water as eluent, followed by $5 \% \mathrm{MeOH}$. The fractions with desalted $\mathrm{N}^{6}$ substituted adenosine nucleotides were pooled, carefully concentrated under reduced pressure, and stored as a 10$\mathrm{mM}$ aqueous stock solution at $-70{ }^{\circ} \mathrm{C}$.

HPLC and HPLC-MS conditions for characterization of synthesized standards

Electrospray ionization (ESI) ${ }^{-}$MS spectra and HPLC purities of the synthesized monophosphates were determined by injecting $10 \mu \mathrm{l}$ of a $10-\mu \mathrm{g} / \mathrm{ml}$ standard solution in the initial mobile phase on a RP Symmetry C18 column $(150 \times 2.1 \mathrm{~mm}, 5 \mu \mathrm{m}$; Waters $)$. The analytes were then separated using a mobile phase consisting of $2 \% \mathrm{~B}$ (methanol) for the first $10 \mathrm{~min}$, followed by a linear gradient to $90 \%$ B from 10 to $25 \mathrm{~min}$ then isocratic $90 \% \mathrm{~B}$ from 25 to $35 \mathrm{~min}$ and finally a linear gradient back to $2 \%$ $\mathrm{B}$ from 35 to $45 \mathrm{~min}$, balanced throughout by (A) aqueous $15 \mathrm{mM}$ ammonium formate $\mathrm{pH} 4.0$, at a constant flow rate of $0.25 \mathrm{ml} / \mathrm{min}$. Using post-column splitting $(1: 1)$, the effluent was introduced simultaneously into the PDA detector (scanning range $210-400 \mathrm{~nm}$, with 1.2-nm resolu- tion) and the MS detector (source temperature $100{ }^{\circ} \mathrm{C}$, capillary voltage $-3.0 \mathrm{kV}$, cone voltage $-30 \mathrm{~V}$, desolvation temperature $\left.250{ }^{\circ} \mathrm{C}\right)$. Nitrogen was used both as the desolvation gas $(600 \mathrm{l} / \mathrm{h})$ and cone gas $(50 \mathrm{l} / \mathrm{h})$. HPLC purities were determined from PDA total ion chromatograms.

For monitoring the reaction progress and the purities of the diphosphates and triphosphates, YMC*Gel ODS-A ${ }^{*}$ M (YMC Europe GmbH, Dinslaken, Germany) C18-120, $11 \mu \mathrm{m}$, in a $250 \times 4 \mathrm{~mm}$ stainless steel column was used as a stationary phase. The eluent consisted of $16 \% \mathrm{ACN}$, $25 \mathrm{mM} \mathrm{Na}_{2} \mathrm{HPO}_{4}$, and $4 \mathrm{mM}$ tetrabutylammonium sulfate $(\mathrm{pH} 7)$ at a flow rate of $1.25 \mathrm{ml} / \mathrm{min}$. Preparative HPLC was accomplished using $\mathrm{YMC}^{*}$ Gel ODS-AQ ${ }^{\mathrm{TM}}$ (YMC Europe $\mathrm{GmbH} /$ Dinslaken/Germany) C18-100, 16- $\mu \mathrm{m}$ material in a $220 \times 50-\mathrm{mm}$ stainless steel column and Q Sepharose FF (Amersham Biosciences/Freiburg/Germany) 90- $\mu \mathrm{m}$ resin in a $125 \times 25 \mathrm{~mm}$ glass column, with a mobile-phase flow rate of $10 \mathrm{ml} / \mathrm{min}$. Mass spectra of the diphosphorylated and triphosphorylated reaction products were acquired by ESI$\mathrm{MS}^{-}$in isopropanol $/ \mathrm{H}_{2} \mathrm{O} / \mathrm{HCOOH}$ (50:49.9:0.1, $\left.v / v / v\right)$ under the following conditions: scan mode, standard normal; ion source type, ESI; flow, $4 \mu \mathrm{l} / \mathrm{min}$; nebulizer gas pressure, $15 \mathrm{psi}$; drying gas flow, $6 \mathrm{l} / \mathrm{min}$; dry temperature, $300{ }^{\circ} \mathrm{C}$; range, $15-1,200 \mathrm{~m} / \mathrm{z}$; capillary voltage, $\pm 4 \mathrm{kV}$; end plate offset, $\pm 0.5 \mathrm{kV}$.

Cell culture

Leukemia cell line K-562 was cultured in standard cell culture medium (DMEM with $5 \mathrm{~g} / 1$ glucose, $2 \mathrm{mM}$ glutamine, $10 \%$ fetal calf serum, $100 \mathrm{U} / \mathrm{ml}$ penicillin, and $100 \mu \mathrm{g} / \mathrm{ml}$ streptomycin). When the cell cultures were in mid-logarithmic growth phase, they were treated with either $10 \mu \mathrm{M}$ iPR (for identification) or the DMSO carrier or left untreated (for validation). The final concentration of DMSO in the medium was $0.1 \%$. After $12 \mathrm{~h}$, the cells were harvested by centrifugation and washed twice in an excess of ice-cold phosphate-buffered saline. The cell suspension was aliquoted (at $10^{6}$ cells per tube for method validation or $5 \times 10^{6}$ cells per tube for identification), collected by 
centrifugation, and flash frozen in liquid nitrogen. All centrifugation steps were done using a cooled centrifuge $\left(4{ }^{\circ} \mathrm{C}\right)$ at $500 \times g$ for $5 \mathrm{~min}$. Samples were stored at $-80{ }^{\circ} \mathrm{C}$ before use.

\section{Sample preparation}

To identify intracellular nucleotides, samples were prepared by the method of Friedecky et al. [20] with slight modifications. iPR-treated cells $\left(5 \times 10^{6}\right.$, for identification and quantification of individual nucleotide forms) or nontreated cells $\left(10^{6}\right.$, for validation) were extracted in $100 \mu \mathrm{l}$ of ice-cold 0.1 M TCA with short ultrasonication (30 s). Samples were left on ice (10 $\mathrm{min}$ ) for deproteination; then, after centrifugation ( $3 \mathrm{~min}, 12,100 \times g)$, the supernatant was collected and back-extracted with diethyl ether $(3 \times 1 \mathrm{ml})$. The water phase was lyophilized to dryness, redissolved in the initial mobile phase, and analyzed by HPLC-MS/MS (for identification) or HPLC-MS (for validation).

\section{Identification of intracellular metabolites by LC-MS/MS}

For MS/MS identification of intracellular metabolites of iPR in treated K-562 cells, samples were dissolved in $60 \mu \mathrm{l}$ of the initial mobile phase $(20 \mathrm{mM}$ ammonium acetate pH 10.0/methanol; 98:2), and $30 \mu \mathrm{l}(50 \%$ of the total sample volume) was injected on an RP column (GeminiNX, $150 \times 2 \mathrm{~mm}, 3 \mu \mathrm{m}$; Phenomenex, Torrance, CA). The analytes were then separated using a mobile phase consisting of $2 \% \mathrm{~B}$ (organic modifier, methanol) for the first $2 \mathrm{~min}$, followed by a linear gradient to $65 \% \mathrm{~B}$ from 2 to $25 \mathrm{~min}$, then isocratic $90 \%$ B from 25 to $35 \mathrm{~min}$, and finally re-equilibration with $2 \%$ $\mathrm{B}$ from 35 to $45 \mathrm{~min}$, balanced throughout by (A) aqueous $20 \mathrm{mM}$ acetic acid adjusted to $\mathrm{pH} 10.0$ with ammonium hydroxide, at a constant flow rate of $0.2 \mathrm{ml} / \mathrm{min}$. ESI MS/MS conditions were set as follows: source temperature $100{ }^{\circ} \mathrm{C}$, capillary voltage $-5.0 \mathrm{kV}$, cone voltage $-33 \mathrm{~V}$, collision energy $25 \mathrm{eV}$, and desolvation temperature $350{ }^{\circ} \mathrm{C}$. Nitrogen was used as the desolvation gas $(400 \mathrm{l} / \mathrm{h})$. The mass spectrometer was operated in product ion scan mode.

Validation of the LC-MS quantification method, quantification of individual nucleotide forms by LC-MS in iPR-treated cells

A sample consisting of $10^{6}$ non-treated (blank) cells was spiked prior to extraction with a known amount of deuterium-labeled and unlabeled standards (10 and $100 \mathrm{pmol}$ authentic, 20 pmol ${ }^{2}$ H-labeled). The cells were then processed as described above. The retention time stability, limit of detection (LOD), linearity, recovery, and analytical accuracy for each analyte were evaluated. Validation was performed under chromatographic conditions used for intracellular metabolite identification (see above). MS conditions (capillary voltage and cone voltage) were optimized to maximize the sensitivity. The effluent was directly introduced into the electrospray source, and the analytes were quantified by negative $\mathrm{ESI}^{-}$selected ion monitoring (SIM) analysis of the deprotonated quasimolecular ion [M$\mathrm{H}]^{-}$. Analyte concentrations were calculated by the isotope dilution method. Due to the lack of deuterium-labeled standards, to quantify different nucleotides, the corresponding trans-zeatin tracers were used (Table 3). The column was thermostatically maintained at $20{ }^{\circ} \mathrm{C}$ to maximize the stability of the retention times.

This method was subsequently used for quantification of individual nucleotide forms in iPR-treated cells $(10 \mu \mathrm{M}$ iPR for $12 \mathrm{~h})$.

\section{Stability experiment}

Stability study of selected analytes (iPTP, iPDP, and iPMP) was performed to monitor eventual hydrolysis of the phosphate groups during sample preparation or measurement. Stabilities were assessed at two different temperatures ( $4{ }^{\circ} \mathrm{C}$ or ambient temperature) and $\mathrm{pH}$ values (initial mobile phase ( $\mathrm{pH} 10.0)$ and/or $0.1 \mathrm{M}$ TCA back-extracted with diethyl ether) within $24 \mathrm{~h}$. Measurements were performed at three time points: 0, 6, and $24 \mathrm{~h}$. Samples were subsequently measured by LC-MS (SIM) method developed.

\section{Results and discussion}

Due to lack of commercially available cytokinin nucleotide standards, various cytokinin nucleotides were prepared (in case of diphosphates and triphosphates for the first time), and their identity and sufficient purity were confirmed by ESI $^{-}$MS, UV, and/or NMR spectroscopy, respectively. The results of physicochemical analyses of the prepared compounds are given in Table 2, along with the reaction conditions. ${ }^{1} \mathrm{H}$ - and ${ }^{13} \mathrm{C}$ - or ${ }^{31} \mathrm{P}-\mathrm{NMR}$ data for prepared compounds were also recorded (data not shown).

The aim of optimizing the HPLC conditions was to achieve baseline separation of nine isoprenoid $\mathrm{CK} 5^{\prime}$ monophosphates, diphosphates, and triphosphates. The $\mathrm{pKa}$ values of the adenosine nucleotides (ATP, ADP, and AMP) are $7.68,7.20$, and 6.67 , respectively [21]. The exocyclic amino group of the adenine exhibits two dissociation constants $\left(\mathrm{pKa}_{1}=4.12, \mathrm{pKa}_{2}=9.83\right)$. Adenine therefore acts as a monovalent anion above $\mathrm{pH} 10.0$. [22]. This positive $\mathrm{p} K \mathrm{a}$ varies slightly with different isoprenoid substitutions [17]. Cytokinin nucleotides thus act as multiple charged anions at $\mathrm{pH}>9.8$. These observations indicate that a basic buffer is appropriate for the separation of CK nucleotides, although they are considered to be acids. The use of a basic 
Table 3 Selected parameters obtained from the HPLC-MS method validation

\begin{tabular}{|c|c|c|c|c|c|c|c|c|c|}
\hline \multirow[t]{2}{*}{ Compound } & \multirow[t]{2}{*}[\mathrm{M}-\mathrm{H}]{$^{-}$} & \multirow[t]{2}{*}{ Internal tracer } & \multirow[t]{2}{*}[\mathrm{M}-\mathrm{H}]{$^{-}$} & \multirow[t]{2}{*}{ RT RSD (\%) } & \multirow[t]{2}{*}{ LOD (pmol) } & \multicolumn{2}{|c|}{ Recovery (\%) } & \multicolumn{2}{|c|}{ Analytical accuracy $(\%)$} \\
\hline & & & & & & $10 \mathrm{pmol}$ & 100 pmol & $10 \mathrm{pmol}$ & $100 \mathrm{pmol}$ \\
\hline iPMP & 414 & ${ }^{2} \mathrm{H}_{5}$-tZRMP & 435 & 0.20 & 0.2 & $97 \pm 4$ & $89 \pm 2$ & $92 \pm 3$ & $94 \pm 1$ \\
\hline iPDP & 494 & ${ }^{2} \mathrm{H}_{5}$-tZRDP & 515 & 0.25 & 0.3 & $91 \pm 3$ & $82 \pm 1$ & $86 \pm 5$ & $61 \pm 1$ \\
\hline iPTP & 574 & ${ }^{2} \mathrm{H}_{5}$-tZRTP & 595 & 0.22 & 0.6 & $81 \pm 2$ & $79 \pm 1$ & $82 \pm 3$ & $90 \pm 1$ \\
\hline tZRMP & 430 & ${ }^{2} \mathrm{H}_{5}$-tZRMP & 435 & 0.24 & 0.2 & $92 \pm 2$ & $88 \pm 1$ & $90 \pm 1$ & $96 \pm 1$ \\
\hline tZRDP & 510 & ${ }^{2} \mathrm{H}_{5}$-tZRDP & 515 & 0.28 & 0.5 & $87 \pm 3$ & $93 \pm 2$ & $86 \pm 3$ & $70 \pm 1$ \\
\hline tZRTP & 590 & ${ }^{2} \mathrm{H}_{5}$-tZRTP & 595 & 0.37 & 0.5 & $80 \pm 2$ & $71 \pm 1$ & $82 \pm 3$ & $81 \pm 1$ \\
\hline cZRMP & 430 & ${ }^{2} \mathrm{H}_{5}$-tZRMP & 435 & 0.23 & 0.5 & $91 \pm 3$ & $83 \pm 1$ & $89 \pm 3$ & $92 \pm 1$ \\
\hline cZRDP & 510 & ${ }^{2} \mathrm{H}_{5}$-tZRDP & 515 & 0.29 & 0.5 & $84 \pm 3$ & $76 \pm 1$ & $82 \pm 4$ & $58 \pm 1$ \\
\hline cZRTP & 590 & ${ }^{2} \mathrm{H}_{5}$-tZRTP & 595 & 0.24 & 0.6 & $79 \pm 1$ & $70 \pm 1$ & $80 \pm 3$ & $79 \pm 1$ \\
\hline
\end{tabular}

buffer precludes the use of a silica-based column since the silica dissolves at $\mathrm{pH}>8$. A hybrid particle column able to sustain basic conditions was used instead, with attention being paid to retention time stabilities.

The separation of nucleotides is probably linked to their polarity increasing with increasing numbers of phosphate groups, while the retention and further separation of cis-transisomers is mainly due to differences associated with variations in the hydrophobic side chain (Fig. 1). Trans-isomers of all three zeatin phosphates eluted before their cis- counterparts, while iP phosphates showed the longest retention. Analogous elution patterns have been reported by Novák et al. for separations of free bases and ribosides by HPLC-MS [9] and for UHPLC-MS/MS identifications of free bases, ribosides, intact $O$-glucosides, and monophosphates [4].

To achieve MS compatibility, ammonium bicarbonate, acetate, and formate at $\mathrm{pH} 10.0$ were tested and compared as additives. Ammonium bicarbonate gave the highest separation efficiencies, but cis- and trans-isomers could not be separated in the 5-20 $\mathrm{mM}$ concentration range using this compound. Therefore, ammonium acetate $(20 \mathrm{mM})$ was selected for further experiments as it showed the essential capability of resolving the cis-and trans-forms of zeatin-type nucleotides (Fig. 1).

The chromatographic performance of RP columns using ion-pairing agents usually declines after 100-300 injections $[23,24]$. During method development, several ion-pairing agents were tested, as well as a variety of commercially available columns. In all cases, the performance declined after 200 injections. Contamination of the MS source was also associated with ion-pairing chromatography (data not shown). However, to date, more than 500 injections have been made on a Gemini-NX column using high $\mathrm{pH}$ buffer, without significant loss of retention or resolution. High $\mathrm{pH}$ chromatography was also found to be more robust than ionpairing chromatography for analyzing adenosine nucleotides analogs. Retention time stabilities determined from 12

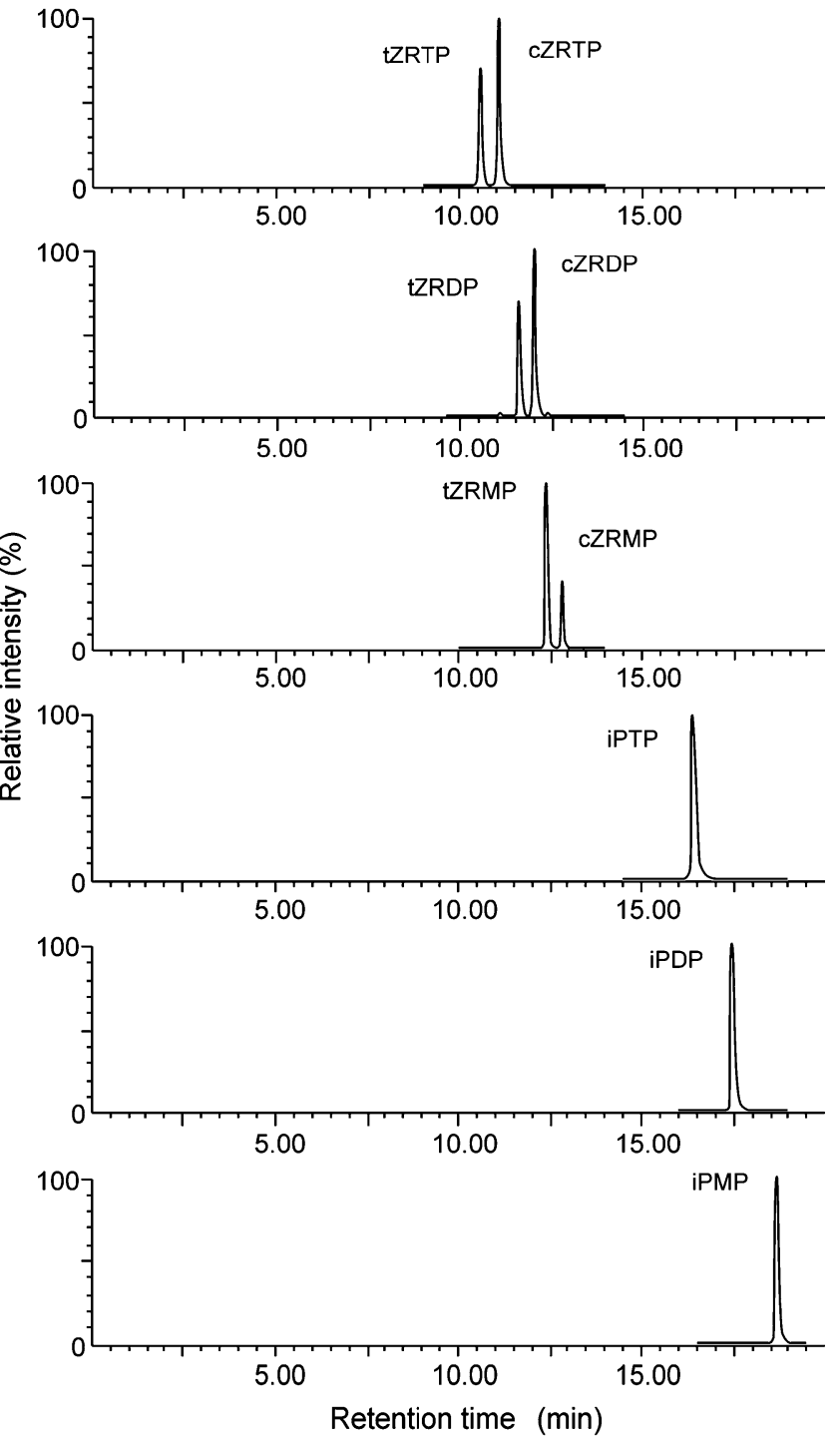

Fig. 1 Reconstructed SIM chromatograms of CK nucleotides used in this study (see Table 1) under optimal chromatographic conditions. Stationary phase: Gemini-NX, $150 \times 2.0 \mathrm{~mm}, 3 \mu \mathrm{m}$. Mobile phase A: $20 \mathrm{mM}$ ammonium acetate $\mathrm{pH} 10.0$; mobile phase B: methanol 

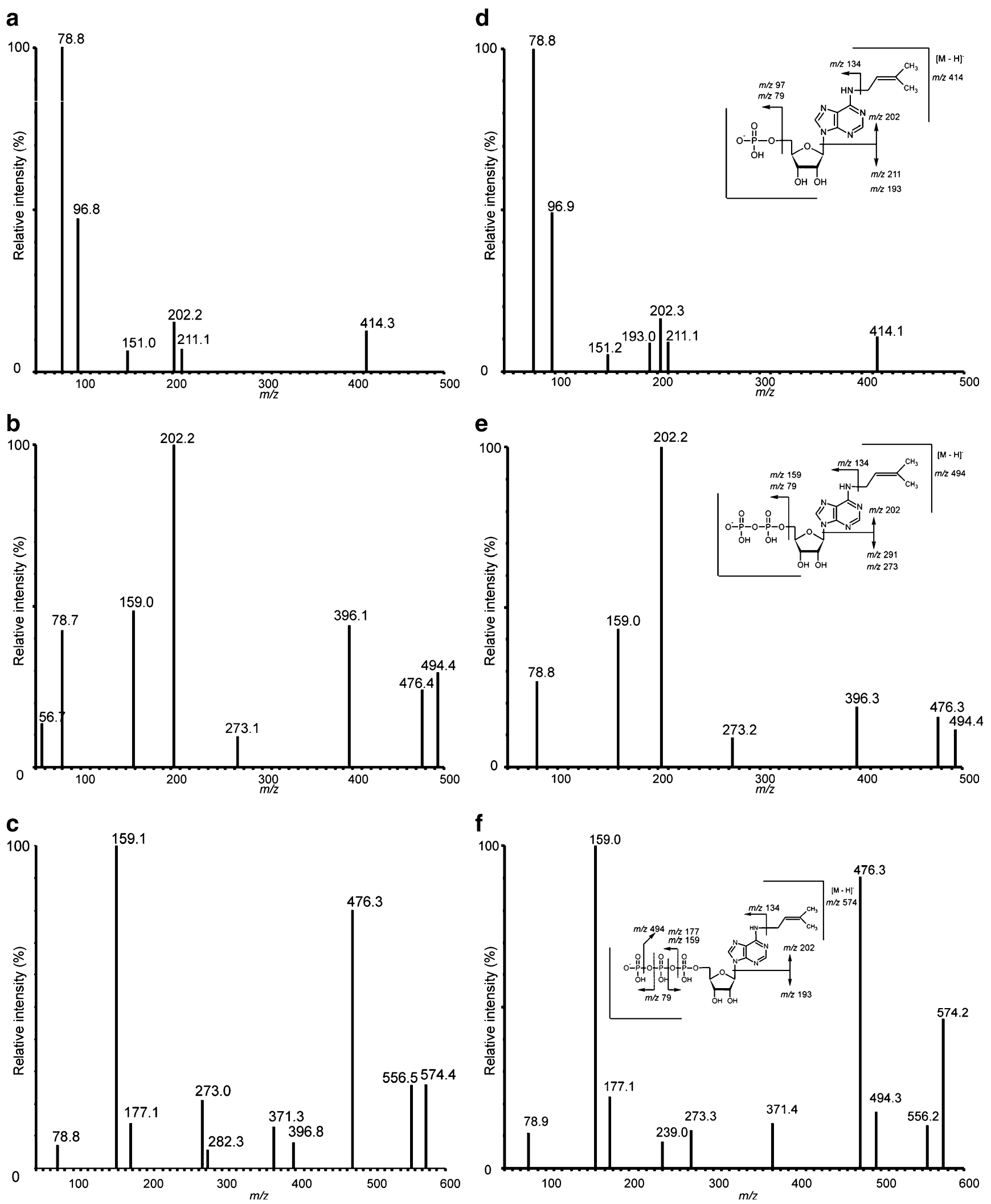

Fig. 2 MS/MS spectra and fragmentation patterns obtained for the putative intracellular metabolites of iPR, a iPMP, $\mathbf{b}$ iPDP, and $\mathbf{c}$ iPTP, extracted from treated cells and the standard solutions of iPMP (d), iPDP (e), and iPTP (f) 
Table 4 Calibration equations and parameters obtained for the nine investigated cytokinin nucleotides $(n=4)$

\begin{tabular}{lllc}
\hline Compound & Calibration equation & $R^{2}$ & Dynamic range (pmol) \\
\hline iPMP & $y=1.13( \pm 0.03) x+0.09( \pm 0.20)$ & 0.996 & $1-1,000$ \\
iPDP & $y=1.15( \pm 0.04) x+0.15( \pm 0.11)$ & 0.997 & $1-1,000$ \\
iPTP & $y=1.25( \pm 0.11) x-0.03( \pm 0.07)$ & 0.998 & $1-1,000$ \\
tZRMP & $y=1.12( \pm 0.02) x+0.10( \pm 0.11)$ & 0.996 & $1-1,000$ \\
tZRDP & $y=1.11( \pm 0.02) x-0.16( \pm 0.05)$ & 0.997 & $1-1,000$ \\
tZRTP & $y=1.15( \pm 0.03) x-0.09( \pm 0.09)$ & 0.998 & $1-1,000$ \\
cZRMP & $y=1.14( \pm 0.06) x-0.42( \pm 0.11)$ & 0.997 & $1-1,000$ \\
cZRDP & $y=1.14( \pm 0.05) x-0.09( \pm 0.14)$ & 0.995 & $1-1,000$ \\
cZRTP & $y=1.17( \pm 0.06) x-0.02( \pm 0.14)$ & 0.997 & $1-1,000$ \\
\hline
\end{tabular}

subsequent injections of the standard solution were acceptable (Table 3). Nucleotide ionization can be achieved in both positive and negative ionization mode [24, 25]. Unsurprisingly, CK nucleotides were not protonized in basic conditions, indicating that negative mode is appropriate. The optimum ionization conditions for quantifying all studied compounds were $-3 \mathrm{kV}$ capillary voltage and $-30 \mathrm{~V}$ cone voltage. The product ion scan showed the products of intracellular metabolism of iPR-treated cells to be iPMP, iPDP, and iPTP. The fragmentation patterns of the diphosphorylated (iPDP) and triphosphorylated (iPTP) metabolites were characterized by the loss of the pyrophosphate group detected at $m / z$ 159. The characteristic fragments of the monophosphorylated anabolite (iPMP) were ions at $\mathrm{m} / z 79$ and 97, which correspond to the loss of a phosphate group. The ion at $m / z 202$ (mainly for iPMP and iPDP) corresponds to the isopentenyl adenine base. The MS/MS spectra, as well as the fragmentation patterns of the iPR-in-vitro-derived nucleotides, are shown and compared with those of standards in Fig. 2. Analogous fragmentation patterns of the diphosphorylated and triphosphorylated anabolites, obtained in negative ionization mode, were observed by Pruvost et al. [26].

It was reported lately [27] that the phosphate group of nucleotides can interact with the stainless steel parts of the HPLC-MS instrumentation. We observed the phenomenon of sensitivity loss as well as peak tailing using UHPLCMS/MS instrumentation (multiple reaction monitoring (MRM) mode). No such phenomenon was observed using our HPLC-MS instrumentation (SIM). We suggest that this could be caused by higher surface area of stainless steel of the UHPLC-MS/MS instrument. This fact led us to validate the method on the HPLC-MS instrument (SIM mode). SIM mode was selected over MRM for quantifying cytokinin nucleotide levels since it showed better linearity (data not shown) and surprisingly comparable detection limits.

The precision of the analytical quantification method was tested by spiking non-treated cells with nine unlabeled and three deuterium-labeled standards at two different concentrations (for details, see the validation method).
Overall, the phosphate recovery rates were good but decreased with increasing number of phosphate groups, being $97 \%$ for monophosphates but only $70 \%$ for triphosphates (Table 3). This was probably due to the lower stability of triphosphates and diphosphates compared to monophosphates, which may also account for the lower accuracy of their analytical determinations. However, when a simple stability study of selected analytes (iPTP, iPDP, and iPMP) was performed (at two different temperatures and $\mathrm{pH}$ values, measurements performed at three time points: 0,6 , and $24 \mathrm{~h}$ ), concentrations of the analytes showed no changes within $24 \mathrm{~h}$, neither in the initial mobile phase (pH 10.0) nor in the 0.1 M TCA back-extracted with diethyl ether kept at $4{ }^{\circ} \mathrm{C}$ in the autosampler. Only the samples dissolved in $0.1 \mathrm{M}$ TCA extracted with diethyl ether showed a decrease of iPTP concentration by $3.8 \%$ and iPDP concentration by $2.6 \%$ if kept at ambient temperature for $24 \mathrm{~h}$. Since the sample preparation took minutes and samples were kept at $4{ }^{\circ} \mathrm{C}$ after being lyophilized and reconstituted in the initial mobile phase, it is not likely that the calculated recoveries are affected by the stability of the analytes. However, we assume that somewhat lower recovery of triphosphates and diphosphates from the spiked samples can still cause their lower stability in cell extract. However, because of using labeled internal standards and stable isotope dilution method for compound quantification, this lower recovery will not, in our opinion, compromise accuracy of the quantification results obtained by this method.

The LODs $(S / N=3)$ obtained during LC-MS validation are in high femtomole concentrations. As expected, the LODs were lowest and highest for the monophosphates and triphosphates, respectively (Table 3). This correlates with the reductions in ionization efficiency with increasing number of phosphate groups [16]. Calibration curves were established for all nine studied compounds and showed linearity across a concentration range of 1-1000 pmol (Table 4).

In a recent study, isopentenyl adenosine diphosphates and triphosphates were found to be present in iPR-treated cells, present at trace concentrations or below the LOD of 
the method applied, and their role in triggering cell death was therefore doubted [8]. Our new method gives the ability to determine the levels of these compounds in riboside-treated cells and showed that they represent $<1 \%$ of the total of iP-derived phosphates found in K-562 cells treated with $10 \mu \mathrm{M}$ iPR for $12 \mathrm{~h}$. Although cytokinin riboside diphosphates and triphosphates probably do not play a role in the rapid induction of cell death described for several leukemia cell lines, they may be responsible for other effects (e.g., changes in mitochondrial proteins, inhibition of protein farnesylation, genotoxic stress) observed in the cell lines with high resistance to apoptosis [28-30]. In order to understand their biological significance in cancer cells, a more complex study is needed. The presented method can also find use in identifying intact endogenous cytokinin nucleotides in plants.

\section{Conclusions}

We have developed and validated a method capable of separating intact monophosphorylated, diphosphorylated, and triphosphorylated cytokinin nucleotides by RP-HPLC in MS-compatible conditions, with no need to use an ionpairing agent. As no standards are commercially available, unlabeled as well as deuterium-labeled standards had to be prepared and used for method development and validation. The quantification method was validated by assessing, for each analyte, the linearity, LOD, recovery, and analytical accuracy by spiking untreated cells. The LODs obtained are in high femtomole concentrations, and linear ranges spanned three orders of magnitude. Moreover, its usefulness was documented by analyzing real samples of iPR-treated K-562 human leukemia cells, where levels of different nucleotide forms such as intracellular metabolites were determined and compared. This chromatographic method also has the potential to be the method of choice for identifying endogenous CK nucleotide compounds in plants.

Acknowledgements This work was supported by the Czech Ministry of Education (MSM 6198959216, 1M06030) and by the Grant Agency of the Czech Republic (522/08/H003, 522/08/0920).

\section{References}

1. Mok DWS, Mok MC (2001) Cytokinin metabolism and action. Annu Rev Plant Physiol Plant Mol Biol 52:89-118

2. Kakimoto T (2001) Identification of plant cytokinin biosynthetic enzymes as dimethylallyl diphosphate: ATP/ADP isopentenyl transferases. Plant Cell Physiol 42:677-685

3. Takei K, Sakakibara H, Sugiyama T (2001) Identification of genes encoding adenylate isopentenyl transferase, a cytokinin biosynthesis enzyme, in Arabidopsis thaliana. J Biol Chem 276:2640526410
4. Novák O, Hauserová E, Amakorová P, Doležal K, Strnad M (2008) Cytokinin profiling in plant tissues using ultra-performance liquid chromatography-electrospray tandem mass spectrometry. Phytochemistry 69:2214-2224

5. Ishii Y, Hori Y, Sakai S, Honma Y (2002) Control of differentiation and apoptosis of human myeloid leukemia cells by cytokinins and cytokinin nucleosides, plant redifferentiationinducing hormones. Cell Growth Differ 13:19-26

6. Doležal K, Popa I, Hauserová E, Spíchal L, Chakrabarty K, Novák O, Kryštof V, Voller J, Holub J, Strnad M (2007) Preparation, biological activity and endogenous occurrence of $N$ 6-benzyladenosines. Bioorgan Med Chem 15:3737-3747

7. Lin BB, Hurley MC, Fox IH (1988) Regulation of adenosine kinase by adenosine-analogs. Mol Pharmacol 34:501-505

8. Mlejnek P, Doležel P (2005) Apoptosis induced by N6-substituted derivatives of adenosine is related to intracellular accumulation of corresponding mononucleotides in HL-60 cells. Toxicol In Vitro 19:985-990

9. Novák O, Tarkowski P, Tarkowská D, Doležal K, Lenobel R, Strnad M (2003) Quantitative analysis of cytokinins in plants by liquid chromatography-single-quadrupole mass spectrometry. Anal Chim Acta 480:207-218

10. Dervieux T, Meyer G, Barham R, Matsutani M, Barry M, Boulieu R, Neri B, Seidman E (2005) Liquid chromatography-tandem mass spectrometry analysis of erythrocyte thiopurine nucleotides and effect of thiopurine methyltransferase gene variants on these metabolites in patients receiving azathioprine/6-mercaptopurine therapy. Clin Chem 51:2074-2084

11. Witters E, VanDongen W, Esmans EL, VanOnckelen HA (1997) Ion-pair liquid chromatography electrospray mass spectrometry for the analysis of cyclic nucleotides. J Chromatogr B 694:55-63

12. Luo B, Groenke K, Takors R, Wandrey C, Oldiges M (2007) Simultaneous determination of multiple intracellular metabolites in glycolysis, pentose phosphate pathway and tricarboxylic acid cycle by liquid chromatography-mass spectrometry. J Chromatogr A 1147:153-164

13. Klawitter J, Schmitz V, Klawitter J, Leibfritz D, Christians U (2007) Development and validation of an assay for the quantification of 11 nucleotides using LC/LC-electrospray ionizationMS. Anal Biochem 365:230-239

14. Coulier L, Bas R, Jespersen S, Verheij E, van der Werf MJ, Hankemeier T (2006) Simultaneous quantitative analysis of metabolites using ion-pair liquid chromatography-electrospray ionization mass spectrometry. Anal Chem 78:6573-6582

15. Jansen RS, Rosing H, de Wolf CJF, Beijnen JH (2007) Rapid Commun Mass Spectrom 21:4049-4059

16. Nordström A, Tarkowski P, Tarkowská D, Doležal K, Åstot C, Sandberg G, Moritz T (2004) Derivatization for LC-electrospray ionization-MS: a tool for improving reversed-phase separation and ESI responses of bases, ribosides, and intact nucleotides. Anal Chem 76:2869-2877

17. Ge LY, Yong JWH, Tan SN, Yang XH, Ong ES (2006) Analysis of cytokinin nucleotides in coconut (Cocos nucifera L.) water using capillary zone electrophoresis-tandem mass spectrometry after solid-phase extraction. J Chromatogr A 1133:322-331

18. Voller J, Zatloukal M, Lenobel R, Doležal K, Béreš T, Kryštof V, Spíchal L, Niemann P, Džubák P, Hajdúch M, Strnad M (2010) Anticancer activity of natural cytokinins: a structure-activity relationship study. Phytochemistry 71:1350-1359

19. Summons RE, Duke CC, Eichholzer JV, Entsch B, Letham DS, MacLeod JK, Parker CW (1979) Mass-spectrometric analysis of cytokinins in plant-tissues.2. Quantitation of cytokinins in Zeamays kernels using deuterium labeled standards. Biomed Mass Spectrom 6:407-413

20. Friedecký D, Tomková J, Maier V, Janoštáková A, Prochazka M, Adam T (2007) Capillary electrophoretic method for nucleotide 
analysis in cells: application on inherited metabolic disorders. Electrophoresis 28:373-380

21. Metzler DE (2001) Biochemistry: the chemical reactions of living cells. Harcourt/Academic, San Diego

22. Barták P, Pěchová D, Tarkowski P, Bednář P, Kotouček M, Stránský Z, Vespalec R (2000) Determination of the first dissociation constant of 6-benzylaminopurine - a comparison of methods. Anal Chim Acta 421:221-229

23. Losa R, Sierra MI, Gion MO, Esteban E, Buesa JM (2006) Simultaneous determination of gemcitabine di- and triphosphate in human blood mononuclear and cancer cells by RP-HPLC and UV detection. J Chromatogr B 840:44-49

24. Vela JE, Olson LY, Huang A, Fridland A, Ray AS (2007) Simultaneous quantitation of the nucleotide analog adefovir, its phosphorylated anabolites and 2'-deoxyadenosine triphosphate by ion-pairing LC/MS/MS. J Chromatogr B 848:335-343

25. Crauste C, Lefebvre I, Hovaneissian M, Puy JY, Roy B, Peyrottes S, Cohen S, Guitton J, Dumontet C, Perigaud C (2009) Development of a sensitive and selective LC/MS/MS method for the simultaneous determination of intracellular 1-beta-D-arabinofuranosylcytosine triphosphate (araCTP), cytidine triphosphate (CTP) and deoxycytidine triphosphate (dCTP) in a human follicular lymphoma cell line. J Chromatogr B 877:1417-1425
26. Pruvost A, Becher F, Bardouille P, Guerrero C, Creminon C, Delfraissy JF, Goujard C, Grassi J, Benech H (2001) Direct determination of phosphorylated intracellular anabolites of stavudine (d4T) by liquid chromatography tandem mass spectrometry. Rapid Commun Mass Spectrom 15:1401-1408

27. Asakawa Y, Tokida N, Ozawa C, Ishiba M, Tagaya O, Asakawa N (2008) Suppression effects of carbonate on the interaction between stainless steel and phosphate groups of phosphate compounds in high-performance liquid chromatography and electrospray ionization mass spectrometry. J Chromatogr A 1198:80-86

28. Cabello CM, Bair WB, Ley S, Lamore SD, Azimian S, Wondrak GT (2009) The experimental chemotherapeutic N6-furfuryladenosine (kinetin-riboside) induces rapid ATP depletion, genotoxic stress, and $\mathrm{CDKN} 1 \mathrm{~A}(\mathrm{p} 21)$ upregulation in human cancer cell lines. Biochem Pharmacol 77:1125-1138

29. Laezza C, Notarnicola M, Caruso MG, Messa C, Macchia M, Bertini S, Minutolo F, Portella G, Fiorentino L, Stingo S, Bifulco M (2006) N6-isopentenyladenosine arrests tumor cell proliferation by inhibiting farnesyl diphosphate synthase and protein prenylation. FASEB J 20:412-418

30. Cheong J, Goh D, Yong JWH, Tan SN, Ong ES (2009) Inhibitory effect of kinetin riboside in human hepatoma, HepG2. Mol Biosyst 5:91-98 